\title{
Morfología, anatomía, ontogenia y composición química de metabolitos secundarios en inflorescencias de Lippia alba (Verbenaceae)
}

\author{
María Isabel Parra-Garcés ${ }^{1}$, José Fernando Caroprese-Araque ${ }^{2}$, Dagoberto Arrieta-Prieto ${ }^{3}$ \& \\ Elena Stashenko ${ }^{4}$ \\ 1,2,3. Laboratorio de Histotecnia, Escuela de Biología, Universidad Industrial de Santander, Facultad de Ciencias, car- \\ rera 27 con Calle 9 Ciudad Universitaria UIS, Bucaramanga, Colombia; Teléfono $(57+7) 6344000$ ext 2354, fax \\ 6349088; marysapgar@gmail.com ${ }^{1}$,josfercar@gmail.com ${ }^{2}$, darrieta@uis.edu.co ${ }^{3}$ \\ 4. Laboratorio de Cromatografía, CIBIMOL-CENIVAM, Universidad Industrial de Santander, Facultad de Ciencias, \\ carrera 27 con Calle 9 Ciudad Universitaria UIS, Bucaramanga, Colombia; Teléfono (57+7) 6344000 ext 2786, fax \\ 6454104; elena@tucan.uis.edu.co
}

Recibido 23-VIII-2009. Corregido 20-VI-2010. Aceptado 26-VII-2010.

\begin{abstract}
Morphology, anatomy, ontogeny and chemical composition of inflorescences volatile secondary metabolites of Lippia alba (Verbenaceae) at three stages of development. There is an increased interest to know and scientifically validate traditional knowledge of medicinal plants. Lippia alba belongs to Verbenaceae family and has been of interest, not only because of its worldwide extensive distribution, but also for its variable use as antiviral, bactericide, citostatic, analgesic and sedative. To study this, the morphology and ontogeny of Lippia alba inflorescences and the chemical composition of its volatile secondary metabolites were analyzed during three different stages of development. Plants were collected at the experimental crop field in CENIVAM, Bucaramanga, Colombia. The inflorescence's morphology and ontogeny, and the chemical composition of volatile secondary metabolites were analyzed using a stereoscopic microscope and chromatographic and spectroscopic techniques. Fresh material corresponding to each stage was fixed in F.A.A (formol, acetic acid and alcohol), included in paraffin and cutted in transversal and longitudinal sections. Sections were stained with safranine-fastgreen, photographed and decribed. The chemical composition of volatile secondary metabolites at each ontogenic stage, was extracted by solid phase micro-extraction in the headspace mode and analyzed by gas chromatography coupled to mass spectrometry. Stage I showed a meristematic mass of cells in vegetative apex and bracts, with an outline of floral whorls. In Stage III, the stamens were adnate, epipetals and didynamous, bicarpelar and syncarpic gynoecium, with superior ovary and decurrent stigma. The main secondary metabolites detected were the bicyclosesquiphellandrene followed by carvone, limonene and trans- $\beta$-farnesene, that constituted the $78 \%$ of the total relative amounts of compounds. Other metabolites such as $\beta$-copaene, $\gamma$-amorphene and $c i s-\beta$-guaiene, were reported for the first time in this study. When compared to other studies, morphological differences reported in this study are possibly related to adaptation to environmental conditions or pollinators, which let us suggest that there is no specific ontogenic pattern. Similarly, the qualitative and quantitative variations in the detected compounds could be explained because one or more of them are used as precursors of others. Rev. Biol. Trop. 58 (4): 1533-1548. Epub 2010 December 01.
\end{abstract}

Key words: Lippia alba, Verbenaceae, inflorescences, floral morphology, anatomy, ontogeny, volatile secondary metabolites.

L. alba (Mill.) N.E. Br., es un arbusto aromático que alcanza los $2 \mathrm{~m}$ de altura. Es erecto, perenne, con hojas pequeñas, opuestas y láminas ovado-oblongas de $2-7 \mathrm{~cm}$, pecioladas y sin estípulas (Yamamoto 2006). El sistema de ramas es homomórfico, es decir, que no se observa especialización en ramas fértiles y vegetativas. En las nuevas ramas que se originan a partir de las yemas prolépticas, las florescencias se desarrollan silépticamente en su conjunto forman las inflorescencias (Múlgura de Romero et al. 1998) y cada una de las flores 
que la conforman producen néctar y polen durante todo el año (Vit et al. 2002). La planta es conocida comúnmente como prontoalivio (Colombia), erva cidreira (Brasil), cidrón (Venezuela), juanilama (Costa Rica) y quitadolor (Centroamérica). Es originaria del bosque seco tropical y subtropical americano (Gupta 1995, Bandoni 2002, Kumar \& Bahl 2002). En Colombia, país donde se realizó esta investigación, se encuentra distribuida en casi todo el territorio hasta los $1800 \mathrm{~m}$ de altitud y con mayor presencia en las regiones del Valle del Cauca, Bolívar, Amazonas, Guajira, Magdalena, Atlántico, Cundinamarca, Meta y Quindío (Guzmán et al. 2004). Se puede encontrar en climas cálido húmedo, cálido seco y templado. Se desarrolla en regiones sin exceso de calor o frío, con temperaturas de hasta $32^{\circ} \mathrm{C}$ con alta intensidad lumínica. Debido a su rusticidad, responde a diversos tipos de suelos como arcillosos y limosos con pH de 5 a 6 (Guzmán et al. 2004). La familia a la cual pertenece (Verbenaceae), incluye cerca de 98 géneros con aproximadamente 2500 táxones específicos (García 1975, Ocampo \& Valverde 2000).

En la comunidad científica, las diversas sinonimias que le son conferidas a $L$. alba (Mill.) N.E. Br., causan confusión (Pascual et al. 2001, Yamamoto 2006). Por esta razón, los estudios de la ontogénesis de la inflorescencia son fundamentales, como lo menciona Mora (1987): "Uno de los capítulos de la morfología de las plantas superiores que permaneció estancado durante siglos, fue sin duda el de la morfología comparativa o tipología de las inflorescencias, no obstante el interés que reviste la comprensión global de estos sistemas para la fisiología, la ecología, la sistemática y la filogenia". Este autor también afirma que "resulta particularmente revelador e instructivo el análisis de diferentes etapas de desarrollo de la estructura en cuestión (ontogenia)". Sin embargo, la publicación de trabajos acerca de morfología y ontogenia floral para taxones del género Lippia es escasa (Rueda 1994).

En los últimos tiempos, L. alba ha despertado gran interés en los investigadores debido a sus propiedades que se constatan con los resultados de diversos estudios. Pino et al. (1996), Zétola et al. (2002) y Oliveira et al. (2006), comprueban la actividad antibacteriana y antifúngica del aceite de esta especie sobre gérmenes humanos de interés clínico. Autores como Vale et al. (1999) y Gurgel et al. (2002) realizaron pruebas con ratones Swiss machos, en las que la acción de componentes del aceite esencial (citral, mirceno y limoneno como compuestos activos) de L. alba es dopaminérgico, causando un efecto depresivo sobre el sistema nervioso central. Carvalho \& Fonseca (2005) mencionan que en el aceite esencial de $L$. alba se encuentra $30-35 \%$ de carvona. Este compuesto tiene diversas aplicaciones, como fragancia y sazonador, inhibidor de la germinación de la papa y semillas de diferentes plantas, agente antimicrobiano y antifúngico, indicador bioquímico ambiental y relevante en el campo médico. Ello justifica el interés en el estudio de este monoterpeno.

Actualmente, sólo el 25\% de las angiospermas han sido estudiadas y algunas familias son totalmente desconocidas en lo concerniente a las esencias florales (Dudareva \& Pichersky 2006). Es así como Evans \& Dickinson (1999), constataron que para examinar hipótesis concernientes a las relaciones entre los miembros de una familia, son necesarios estudios de morfología, ontogenia floral y análisis fitoquímicos, entre otros.

Considerando la permanente búsqueda de nuevos productos y combinaciones de sustancias químicas, se ven impulsados los estudios detallados de la familia Verbenaceae (Stashenko et al. 2004) y, particularmente Lippia, es un género promisorio que amerita su investigación, entre otros, para aislar principios activos nuevos (Pascual et al. 2001).

El presente trabajo es una contribución al cuidadoso estudio que necesita la gran diversidad de flora colombiana y puntualmente, al conocimiento ontogénico, morfológico y anatómico de L. alba, ya que se precisan caracteres del desarrollo floral, los cuales constituyen una herramienta fundamental para posteriores profundizaciones taxonómicas. Igualmente, la determinación de los compuestos químicos 
contenidos en las inflorescencias será útil en investigaciones que pretendan verificar sus efectos sobre el ser humano y demás organismos que los ingieren, pues, "el consumo indiscriminado de esta y otras especies suele ser a menudo "formulado" para actividades contradictorias y sin un control debido en la medicina vernácula, que utiliza principalmente las hojas y las flores" (Pascual et al. 2001 y Ricciardi et al. 2000). Es fundamental entonces, el aval científico derivado de trabajos de esta naturaleza.

Por primera vez, mediante este estudio, se registra en forma simultánea una descripción de la organogénesis floral en esta verbenácea y los compuestos químicos volátiles que se forman durante las tres etapas elegidas de este proceso, llegando a evidenciar los cambios que se dan en la composición de la fragancia a medida que avanza el desarrollo de la inflorescencia.

\section{MATERIALES Y MÉTODOS}

Material vegetal: Para la realización de este trabajo se utilizaron inflorescencias de $L$. alba, que consisten de un eje central que soporta los agrupamientos florales y al igual que una flor, inician en las yemas florales (Mora 1987). El muestreo de las inflorescencias se realizó en las instalaciones de cultivos experimentales del Centro Nacional de Investigaciones para la Agroindustrialización de Especies Vegetales Aromáticas y Medicinales Tropicales (CENIVAM), en época seca (febrero) al medio día, ya que cuando se presentan condiciones de alta radiación solar y temperaturas elevadas, se obtienen contenidos altos de metabolitos secundarios, en especial de carvona (Duarte \& Cárdenas 2007). La determinación del material vegetal se efectuó en el Herbario Nacional Colombiano de la Universidad Nacional de Colombia (Bogotá) donde quedó depositado un espécimen testigo de Lippia alba (COL512078).

El material recolectado fue observado al microscopio estereoscópico para el establecimiento de las tres etapas de desarrollo (entre cada etapa transcurren 15 días aproximadamente), teniendo en cuenta características de los diferentes verticilos florales: tamaño, color y cambios morfológicos (como la presencia y ausencia de las pequeñas corolas que conforman la inflorescencia). Al llegar al tercer grado de desarrollo (flor madura), se realizó la descripción de la morfología y anatomía floral (gineceo, androceo y verticilos en general). Las muestras seleccionadas fueron fijadas en FAA (formol, ácido acético, alcohol), incluidas en parafina y seccionadas en cortes transversales y longitudinales a $10 \mu \mathrm{m}$ con micrótomo rotatorio. Para la tinción se utilizó safranina-fastgreen (Johansen 1940) y la fijación de los cortes a las láminas se hizo con bálsamo de Canadá. Para las observaciones y obtención de fotografías se utilizó un microscopio óptico OLYMPUS, Japón.

Análisis químico: El análisis químico se realizó según la metodología propuesta por Stashenko et al. (2004). Para el proceso de microextracción en fase sólida de la fase vapor (headspace) (HS-SPME) del material vegetal, se usó una fibra de poli(dimetilsiloxano)/divinilbenceno (PDMS/DVB, 65 $\mu \mathrm{m}$ ) obtenida de Supelco Inc. (Bellefonte, PA, EE.UU.). Gases especiales para cromatografía fueron obtenidos de Aga-Fano S.A. (Bucaramanga, Colombia). Se muestreó la fase vapor a $22 \pm 1^{\circ} \mathrm{C}$, de $0.7 \mathrm{~g}$ de planta, colocada en un frasco de $20 \mathrm{ml}$. El tiempo de pre-equilibrio del frasco, fue de $15 \mathrm{~min}$ y el tiempo de exposición de la fibra fue de 40min (la selección de este parámetro se basó en experimentos preliminares). Las sustancias extraídas fueron térmicamente desorbidas a $250^{\circ} \mathrm{C}$, durante $2 \mathrm{~min}$, en un puerto de inyección del cromatógrafo de gases, usando un liner especial para SPME de volumen reducido.

El análisis cromatográfico de las muestras se realizó en un GC Hewlett-Packard (HP) 5890A Series II, equipado con un inyector split/splitless $\left(250^{\circ} \mathrm{C}\right.$, relación split $\left.1: 10\right)$ y un detector de ionización en llama (FID) $\left(250^{\circ} \mathrm{C}\right)$, por triplicado para cada muestra (en total nueve muestras). Los datos cromatográficos fueron adquiridos y procesados con un programa HP Chem Station HP Rev. A.06.03. Para la 
separación de mezclas se usó una columna capilar apolar, HP-5 (Hewlett-Packard) de $30 \mathrm{mx} 0.25 \mathrm{~mm}$, D.I.x0.25 $\mu \mathrm{m}, \mathrm{d}_{\mathrm{f}}$, con fase estacionaria de 5\%-fenil-poli(metilsiloxano). $\mathrm{La}$ temperatura del horno fue programada de $45^{\circ} \mathrm{C}$ (2min) hasta $150^{\circ} \mathrm{C}$ a razón de $5 \mathrm{~min}^{-1}$ para la columna apolar, HP-5 y de $60^{\circ} \mathrm{C}(5 \mathrm{~min})$ hasta $280^{\circ} \mathrm{C}$ a razón de $8^{\circ} \mathrm{C} \min ^{-1}$ para la columna polar INNOWAX. El gas de arrastre empleado fue helio (99.9995\%, Aga-Fano, Bucaramanga S.A,), con una presión de entrada en la cabeza de la columna de 15 psi y una velocidad lineal de $26 \mathrm{~cm} / \mathrm{s}$. El gas auxiliar fue nitrógeno (99.995\%; Aga-Fano, Bucaramanga S.A), con una velocidad de flujo de $30 \mathrm{ml} / \mathrm{min}$. Las velocidades de flujos de gases de combustión en el FID, el aire y el hidrógeno, fueron de 300 y $30 \mathrm{ml} / \mathrm{min}$, respectivamente. Los espectros de masas se obtuvieron por impacto de electrones con energía de 70eV, en un CG Agilent Technologies 6890 Plus acoplado a un detector selectivo de masas Hewlett Packard MSD 5972 (Cromatografía de gases acoplada a espectrometría de masas (GC-MS)), y equipado con un puerto de inyección split/splitless (1:10), un inyector automático Agilent 7863, un sistema de datos HP MS ChemStation (Versión 1.05), incluyendo las bases de datos NBS 75K, WILEY 138K y ADAMS. Se usó una columna capilar de sílice fundida, HP-5MS de 30m x 0.25mm, D.I., con la fase estacionaria de 5\%-fenil-poli(metilsiloxano) de $0.25 \mathrm{~mm}$ de grosor. El gas de arrastre fue helio (99.9995\%, Aga-Fano, Bucaramanga S.A.), con una velocidad lineal de $35 \mathrm{~cm} / \mathrm{s}$. Las temperaturas de la cámara de ionización y de la línea de transferencia se mantuvieron a 230 y $285^{\circ} \mathrm{C}$, respectivamente. Los espectros de masas y corrientes iónicas reconstruidas (TIC) se obtuvieron en un cuadrupolo, por medio de barrido automático de frecuencia (full scan), a $4.75 \mathrm{scan} \mathrm{s}^{-1}$, en el ámbito de masas de $m / z$ 40-350. La homogeneidad de cada pico cromatográfico fue revisada, obteniendo fragmentogramas de masas, basados en iones característicos de cada compuesto. Para la identificación de los compuestos se usaron espectros de masas e índices de retención de componentes de Kovàts (Kovàts 1965) de componentes, que se compararon con los existentes en la literatura (Adams 1995, Joulian \& König 1998).

Los datos fueron recopilados como media \pm error estándar de la media (SEM). Posteriormente, para establecer las diferencias significativas en la variación de los porcentajes relativos de los metabolitos secundarios volátiles de $L$. alba en las tres etapas de desarrollo, se realizó un análisis de varianza (ANOVA) de una vía tipo modelo lineal general (MLG), verificando previamente los supuestos de normalidad (prueba de Kolmogorov-Simirnov) y homogeneidad de varianzas (prueba de Levene). Por la naturaleza de los datos, éstos fueron transformados usando la raíz del arco-seno. Adicionalmente se utilizó la prueba de Tukey para comparar las medias entre las diferentes etapas de desarrollo de todos los metabolitos encontrados. El criterio de significancia fue $p<0.05$. Los análisis estadísticos fueron realizados usando el programa SPSS para Windows, versión 13.0.

\section{RESULTADOS}

Morfología y anatomía floral: Las inflorescencias de L. alba son cabezuelas glomerulares de ubicación axilar, opuestas y en pares (Fig. 1A, B). Las flores pubescentes y sésiles están insertas sobre una parte del receptáculo común sobre un raquis, acompañadas por

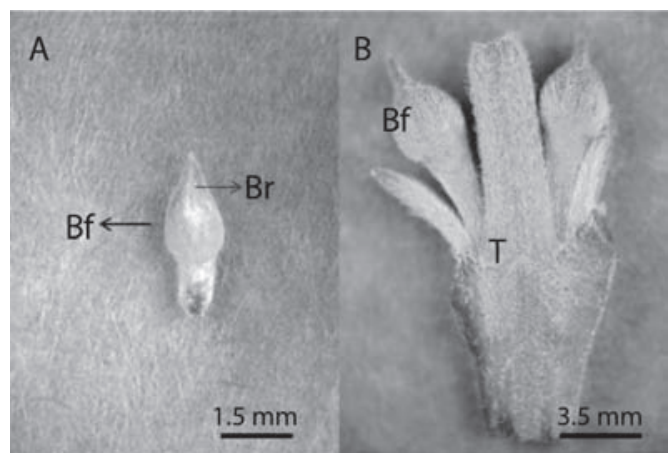

Fig. 1. Botón floral, característico de la Etapa I.Abreviaturas: Bf:Botón floral; Br:Bráctea; T:Tallo.

Fig. 1.Floral buds, characteristic of stage one.Abbreviations: Bf: Floral bud; Br: Bract; T: Stalk. 
brácteas poco diferenciadas (Fig. 1A). La Etapa I corresponde a los botones verdes pubescentes de $c a .1 .5 \mathrm{~mm}$ (Fig. 1B), de disposición acrópeta al tallo, tal como lo observaron Múlgura de Romero et al. (1998).

Microscópicamente, la Etapa I se caracteriza por su mínimo desarrollo. Al inicio de la diferenciación meristemática (Fig. 2A, B, C, D) se aprecia el ápice vegetativo protegido por una bráctea incipiente, y por debajo a la derecha, un primer ápice floral protegido por una bráctea larga. Se observan a derecha e izquierda, por debajo del ápice vegetativo, cuatro primordios en diferenciación dispuestos en verticilos (Fig. 2D) y rodeados por aproximadamente 12 brácteas; Éstas soportarán posteriormente a las flores maduras. Es interesante destacar la presencia de un elevado número de brácteas previo a la aparición de los verticilos de reclamo (Fig.
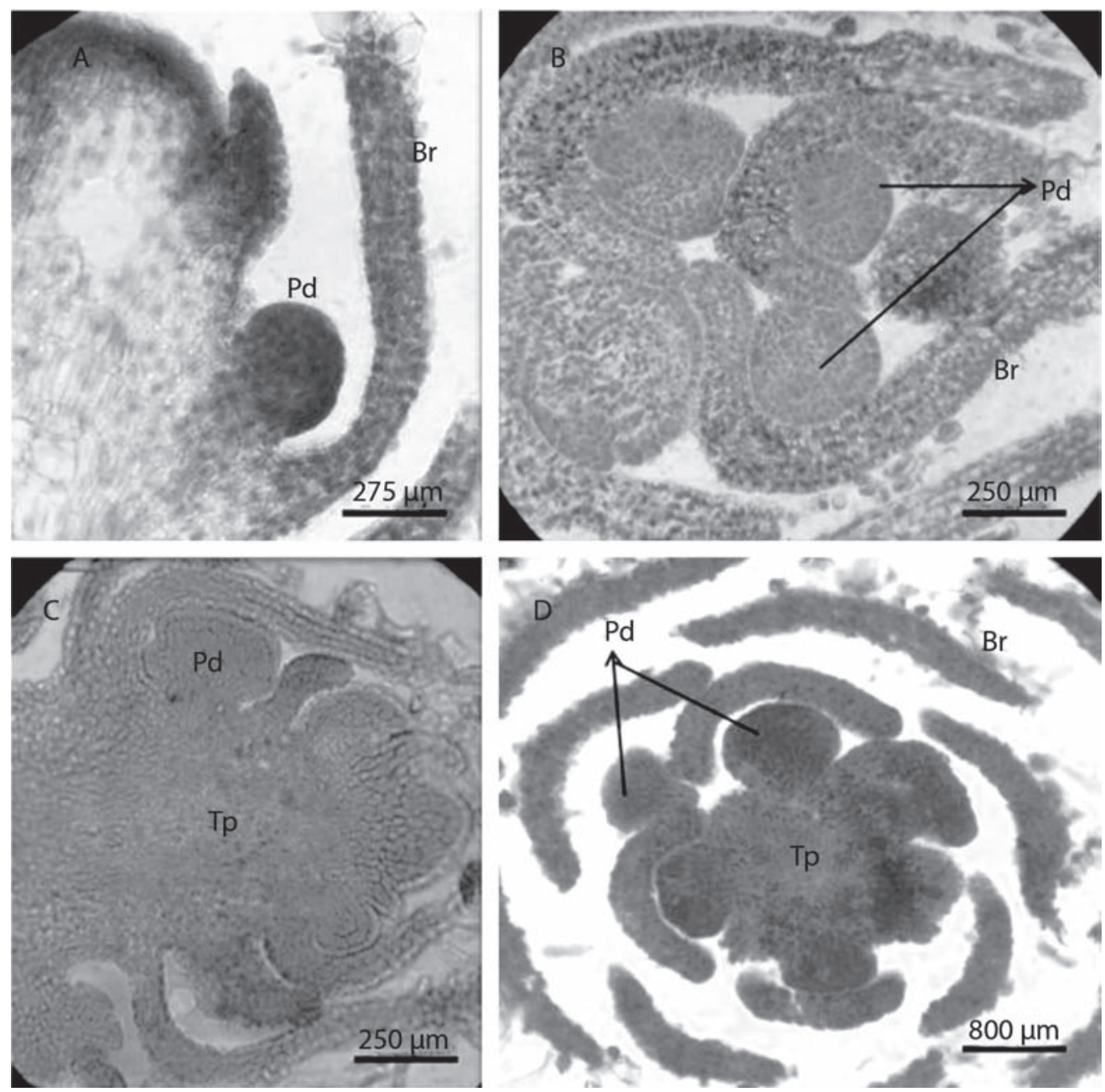

Fig. 2. Cortes longitudinales (A,B,C) y transversal (D) de las inflorescencias de L. alba en la Etapa I. Abreviaturas: Pd:Primordio en diferenciación; Br:Bráctea; Tp:Tejido parenquimático.

Fig. 2. Longitudinal (A,B,C) and cross-sectional cuts (D) of L. alba inflorescence in stage one. Abbreviations: Pd: Primordium in differentiation; Br: Bract; Tp: Parenchymal tissue. 
2A, B). Se insinúa una maduración acrópeta de las flores (Fig. 2B, C).

La Etapa II difiere macroscópicamente de la Etapa anterior, en el tamaño de ca. $2.5 \mathrm{~mm}$ y la presencia de múltiples brácteas más diferenciadas, pubescentes, imbricadas, que no permiten ver a simple vista el desarrollo de estructuras florales (Fig. 3A). En los cortes longitudinales observados al microscopio estereoscópico es notoria la individualización de cada flor y se percibe claramente una maduración acrópeta (Fig. 3B). Una diferencia más notoria con respecto a la Etapa I de desarrollo puede apreciarse a nivel microscópico, donde se observa cómo la acción de procesos morfogenéticos dan paso a la formación de los verticilos florales de reclamo o perianto: cáliz y corola (Fig. 4B, D).

En la Etapa II se puede apreciar la estructura carpelar central que dará origen al gineceo acompañada por los primordios de los futuros estambres (Fig. 4A,C). Se observa de igual manera, que ambos verticilos aún inmaduros, se encuentran protegidos del exterior por la corola, el cáliz y la bráctea (Fig. 4A). La flor presenta un pedicelo bastante reducido y está soportada siempre por su respectiva bráctea (Fig. 4B,C). Se evidencia el inicio de la formación de sépalos, pétalos y estambres desde la base y en forma centrípeta. Es de destacar que los estambres son petalostémonos (Fig. 4A,B).

La Etapa III presenta macroscópicamente una diferencia marcada con respecto a las dos
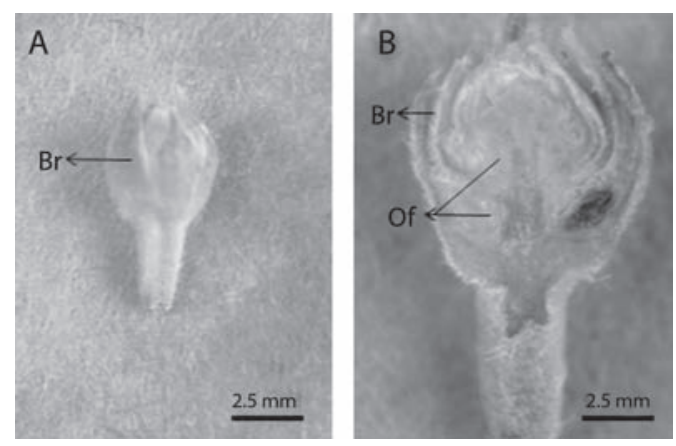

Fig. 3. Botones florales presentes en la Etapa II. Abreviaturas: Br:Brácteas; Of:Órganos en formación.

Fig. 3. Floral buds present in stage two. Abbreviations: Br:Bracts; Of:Organs in formation. anteriores. Se observa por primera vez la inflorescencia de $c a .5 \mathrm{~mm}$ con varias flores pequeñas tubulares de color lila (Fig. 5A) y con todos los órganos florales completamente desarrollados: el gineceo con ovario súpero, sincárpico, bicarpelar, bilocular, con estigma decurrente y amarillo verdoso. El androceo es epipétalo con dos pares de estambres didínamos de color amarillo (Fig. 5B). Las flores son gamopétalas, bilabiadas (zigomorfas), sésiles y pubescentes; Presentan dos sépalos blancos, vellosos y libres (dialisépalos). Abrazando cada par de sépalos hay una bráctea ovoide y verde (Fig. 5B).

Microscópicamente se observan detalles de las estructuras reproductoras. El ovario es bilocular con óvulos anátropos de placentación axial (Fig. 6A, F), el estigma con varios tricomas que le dan una apariencia plumosa (Fig. 6A,B), el androceo es epipétalo, formado por estambres didínamos con filamentos adnados a la corola (petalostémonos) y anteras ditecas libres (Fig. 6C, D, E).

Metabolitos secundarios volátiles: En la Etapa I se detectaron e identificaron en el perfil cromatográfico de los metabolitos secundarios volátiles aislados mediante HS-SPME, 22 compuestos en cantidades relativas $>0.05 \%$ (Fig. 7A). Para la Etapa II se detectaron e identificaron 21 compuestos (Fig. 7B), igual que en la III (Fig. 7C). En la composición química de todas las fases de desarrollo predominaron los sesquiterpenos.

El Cuadro 1 muestra la cantidad relativa $(\% \pm \mathrm{SD}, \mathrm{n}=3)$ de los metabolitos secundarios volátiles aislados por HS-SPME. Todos los metabolitos detectados presentaron diferencias cuantitativas significativas.

Todos los metabolitos identificados se registraron durante el desarrollo de la inflorescencia de L. alba, salvo el $\gamma$-muuroleno, que se detectó en la Etapa I de desarrollo, pero no en las Etapas II y III.

Entre los cuatro principales metabolitos detectados (Cuadro 1, Fig. 7), se encontró biciclosesquifelandreno con un porcentaje relativo del $25 \%$ en la Etapa I, mientras en la Etapa II aumentó a $36.13 \%$ y en la Etapa III 

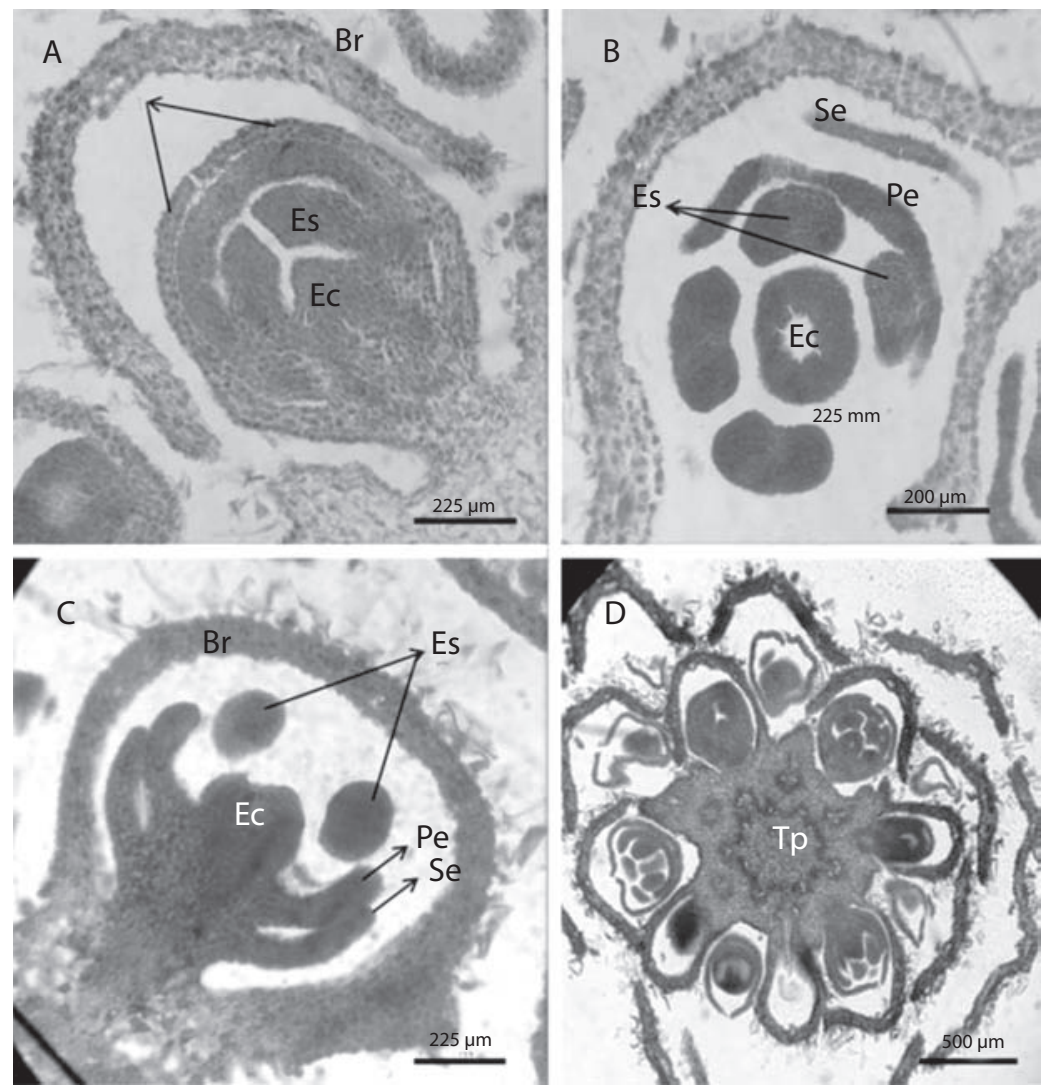

Fig. 4. Cortes longitudinales (A, C) y transversal (B, D) de las inflorescencias de L. alba en la Etapa II. Abreviaturas: Br:Bráctea; Se:Sépalos; Pe:Pétalos; Es:Estambres; Ec:Estructura carpelar; Tp:Tejido parenquimático; Ca:Cáliz; Co:Corola.

Fig. 4. Longitudinals (A, C) and cross-sectional cut (B,D) of L. alba inflorescence in stage two. Abbreviations: Br:Bract; Se:Sepals; Pe:Petals; Es:Stamen; Ec:Carpelar structure; Tp:Parenchymal tissue; Ca:Calyx; Co:Corolla.
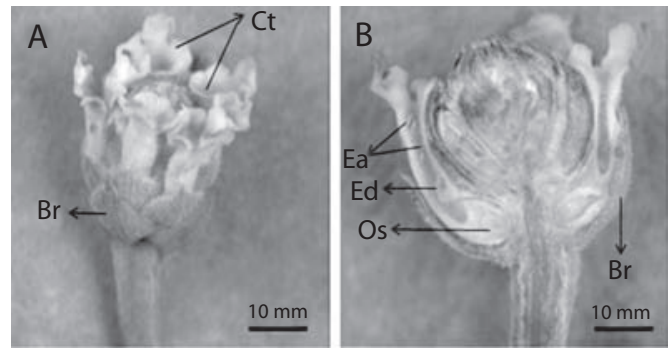

Fig. 5. Inflorescencias en su máximo desarrollo, con flores abiertas correspondientes a la Etapa III. Abreviaturas: Br:Bráctea; Ct:Corola tubular; Ea:Estambres adnados; Ed:Estigma decurrente; Os:Ovario súpero.

Fig. 5. Inflorescences in their maximum development stage (Stage three), with open flowers. Abbreviations: Br:Bract; Ct:Tubulate corolla; Ea:Adnates stamens; Ed:Decurrent stigma; Os:Upper ovary. se incrementó hasta un 38\%. La carvona, con un porcentaje relativo de $23.6 \%$ en la Etapa I, mientras en la Etapa II aumentó hasta llegar a $27 \%$ y en la Etapa III se incrementó hasta 29.14\%. El metabolito secundario limoneno, registró un $4.7 \%$ en la Etapa I y disminuyó hasta $3.2 \%$ durante la Etapa III. Por último, el trans- $\beta$-farneseno, exhibió un porcentaje de $4.6 \%$ en la Etapa I, disminuyó en la Etapa II a $3.7 \%$ y llegó a $1.2 \%$ en la Etapa III.

\section{DISCUSIÓN}

En este estudio morfológico, anatómico y ontogenético realizado para $L$. alba, se observó un estigma decurrente y con tricomas 

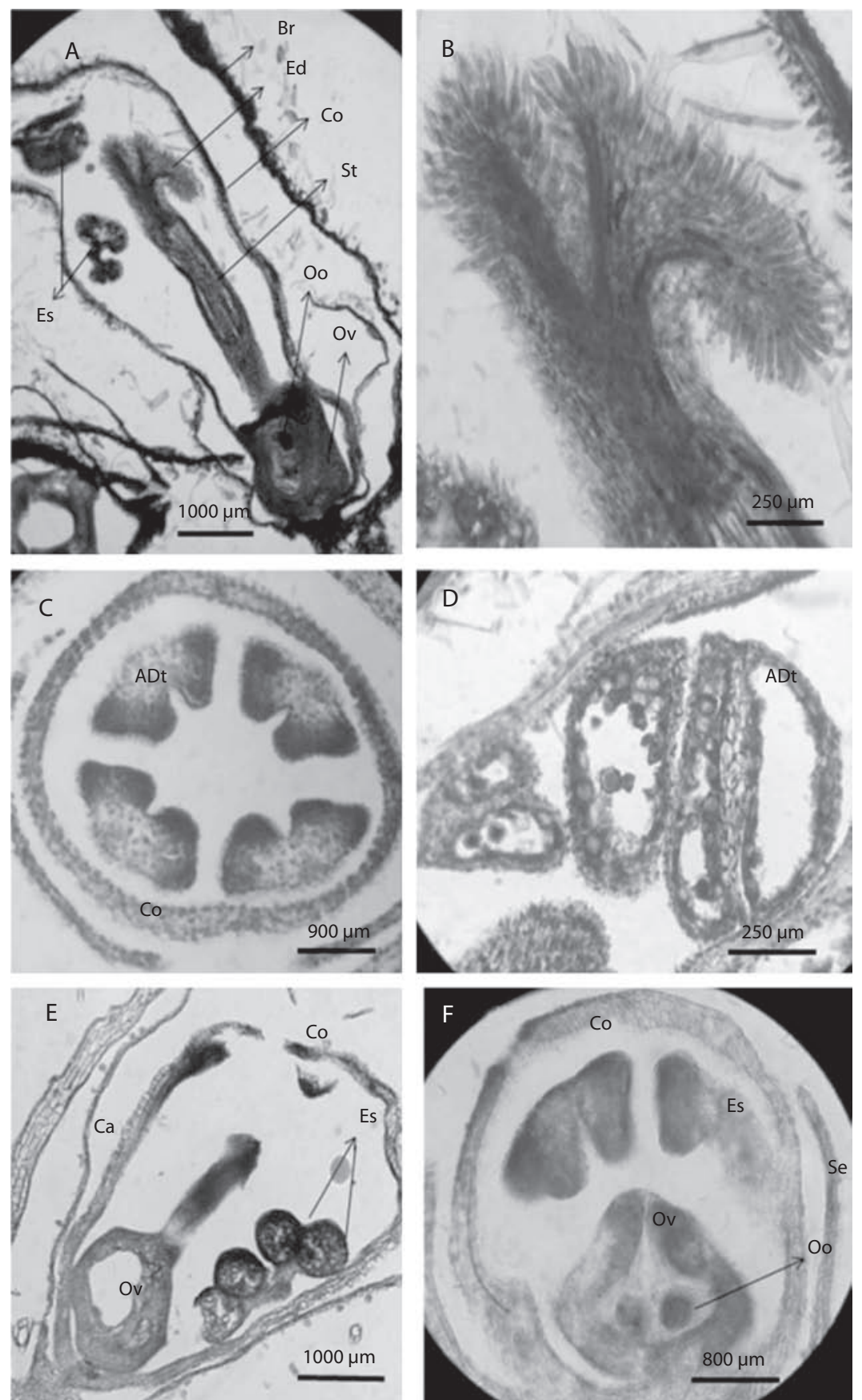

Fig. 6. Cortes longitudinales (A,B,D,E,F) y transversal (C) de las inflorescencias de L. alba en la etapa III. Abreviaturas: Br:Bráctea; Es: Estambres; Ed:Estigma decurrente; Se:Sépalos; Oo:Óvulo; Ov:Ovario; Co:Corola; St:Estilo; Ca:Cáliz; $\mathrm{ADt}$ :Anteras ditecas.

Fig. 6. Longitudinal (A,B,D,E,F) and cross-sectional cut (C) of L. alba inflorescence in stage three. Abbreviations: Br:Bract; Es:Stamens; Ed:Decurrent stigma; Se:Sepals; Oo:Ovule; Ov:Ovary; Co:Corolla; St:Style; Ca:Calyx; ADt:Dithecals anthers. 


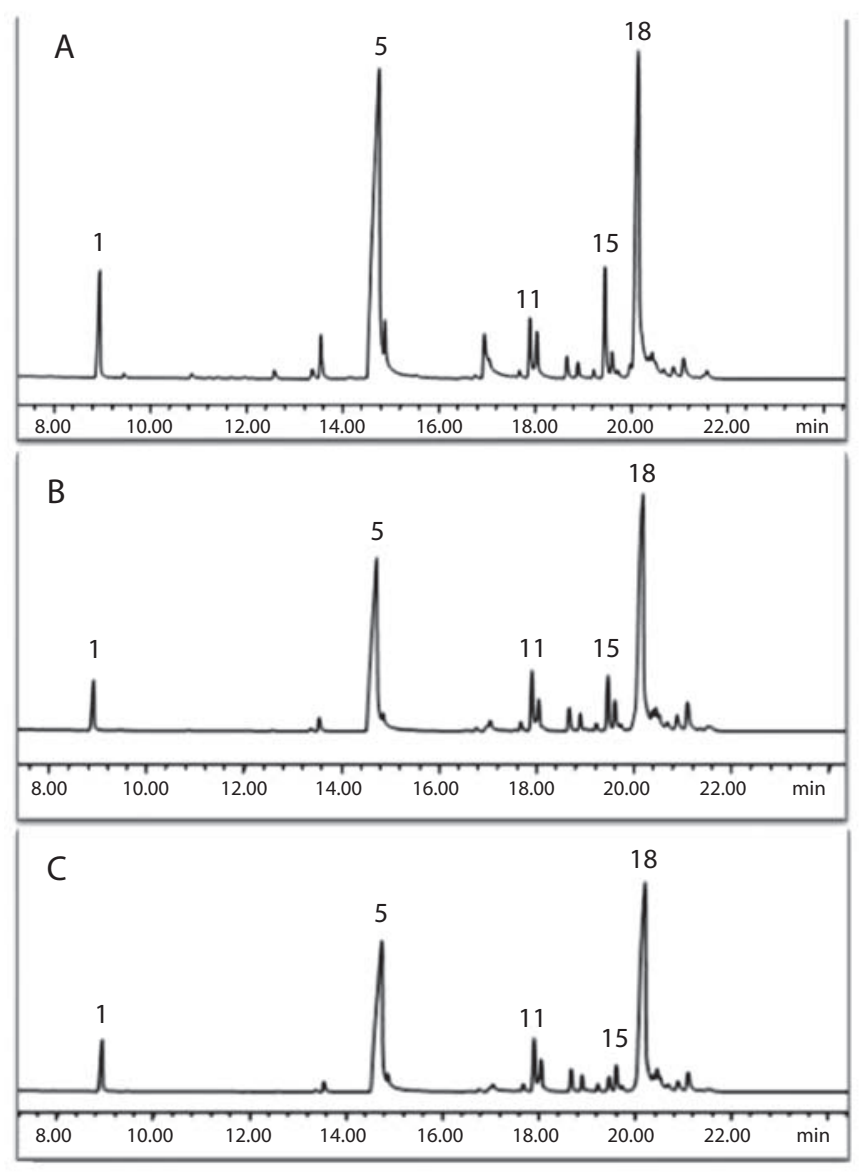

Fig. 7. Cromatogramas (CG-MS) de los metabolitos secundarios volátiles de las inflorescencias de L. alba, obtenidos por HS-SPME. A) Etapa I, B) Etapa II, C) Etapa III. La identificación de los picos aparece en el cuadro 1.

Fig. 7. Volatile secondary metabolites chromatograms (CG-MS) from inflorescences of L. alba, extracted by HS-SPME. A) Stage one, B) Stage two, C) Stage three. Peaks identified in table 1.

(apariencia plumosa), y no capitado, como lo afirman Muñoz et al. (2007). Esta podría ser una variación que obedece a adaptaciones a condiciones ambientales o quizá a polinizadores. Al respecto Cerana (2004) señala que para la familia Asteraceae, por ejemplo, la disposición del estigma puede variar para ser más receptivo a la polinización cuando la flor es visitada por un insecto en cierta época. Perreta \& Vegetti (2004), mencionan la variación observada en la longitud de las inflorescencias que podría deberse a factores ambientales y a la menor o mayor plasticidad morfológica. Dong et al. (2005) y Krizek \& Fletcher (2005), coinciden en que los mecanismos moleculares contribuyen a la identidad y posición de los órganos florales, a la extrema variación en las morfologías florales y a su vez a una relativa simplicidad de las estructuras. Todas estas diferencias nos permiten ver que no hay un patrón específico para el desarrollo floral en algunas familias de dicotiledóneas y que estas características podrían apoyar estudios filogenéticos vegetales. 


\section{CUADRO 1}

Promedio $\pm S D$ de cantidad relativa de metabolitos volátiles en $\mathrm{L}$. alba durante tres etapas de desarrollo ontegénico, número de pico e índices de Kovats. Significancia según test de Anova, letras diferentes $(a, b, c)$

indican diferencias significativas, según el test de Tukey $(p<0.05)$

TABLE 1

Average relative quantitive $( \pm S D)$ of volatile metabolites in $\mathrm{L}$. alba during three stages of ontogenic development, number of peaks and Kovats index. Significance according to Anova test; different letters $(a, b, c)$ indicate significant differences, according to the test Tukey $(p<0.05)$

\begin{tabular}{|c|c|c|c|c|c|c|c|}
\hline \multirow{2}{*}{ No pico } & \multirow{2}{*}{ Ik } & \multirow{2}{*}{ Tipo ${ }^{x)}$} & \multirow{2}{*}{ Compuesto } & \multicolumn{4}{|c|}{ Cantidad relativa, $\%$} \\
\hline & & & & E1 & E2 & E3 & $\mathrm{p}$ \\
\hline 1 & 1030 & $\mathrm{M}$ & Limoneno & $4.70 \pm 0.14 \mathrm{a}$ & $3.80 \pm 0.03 \mathrm{~b}$ & $3.20 \pm 0.05 \mathrm{c}$ & $\mathrm{p}<0.001 * * *$ \\
\hline 2 & 1169 & MO & Borneol* & $0.40 \pm 0.05 \mathrm{a}$ & $1.40 \pm 0.06 \mathrm{~b}$ & $1.80 \pm 0.09 \mathrm{c}$ & $\mathrm{p}<0.001 * * *$ \\
\hline 3 & 1199 & MO & cis-Dihidro Carvona & $0.53 \pm 0.04 \mathrm{a}$ & $0.24 \pm 0.09 \mathrm{~b}$ & $0.23 \pm 0.07 \mathrm{~b}$ & $\mathrm{p}<0.01 * *$ \\
\hline 4 & 1207 & MO & trans-Dihidro Carvona & $1.80 \pm 0.09 \mathrm{a}$ & $2.70 \pm 0.08 \mathrm{~b}$ & $0.85 \pm 0.05 \mathrm{c}$ & $\mathrm{p}<0.001 * * *$ \\
\hline 5 & 1256 & MO & Carvona & $23.60 \pm 0.43 \mathrm{a}$ & $27.0 \pm 0.20 \mathrm{~b}$ & $29.14 \pm 0.11 \mathrm{c}$ & $\mathrm{p}<0.001 * * *$ \\
\hline 6 & 1261 & MO & Piperitona & $3.41 \pm 0.31 \mathrm{a}$ & $2.01 \pm 0.04 \mathrm{~b}$ & $2.50 \pm 0.07 \mathrm{ab}$ & $\mathrm{p}<0.001 * * *$ \\
\hline 7 & 1343 & MO & Piperitenona & $2.70 \pm 0.06 \mathrm{a}$ & $0.34 \pm 0.11 \mathrm{~b}$ & $0.41 \pm 0.06 \mathrm{c}$ & $\mathrm{p}<0.001 * * *$ \\
\hline 8 & 1348 & SE & $\alpha$-Cubebeno* & $2.30 \pm 0.24 \mathrm{a}$ & $1.20 \pm 0.07 \mathrm{~b}$ & $0.94 \pm 0.05 \mathrm{c}$ & $\mathrm{p}<0.001 * * *$ \\
\hline 9 & 1380 & SE & $\alpha$-Copaeno & $0.40 \pm 0.10 \mathrm{a}$ & $0.61 \pm 0.14 \mathrm{~b}$ & $0.51 \pm 0.05 \mathrm{ab}$ & $\mathrm{p}<0.01 * *$ \\
\hline 10 & 1389 & SE & $\beta$-Bourboneno & $2.50 \pm 0.16 \mathrm{a}$ & $3.13 \pm 0.04 \mathrm{~b}$ & $3.70 \pm 0.07 \mathrm{c}$ & $\mathrm{p}<0.001 * * *$ \\
\hline 11 & 1395 & SE & $\beta$-Elemeno & $2.54 \pm 0.13 \mathrm{a}$ & $1.40 \pm 0.06 \mathrm{~b}$ & $2.30 \pm 0.10 \mathrm{ab}$ & $\mathrm{p}<0.01 * *$ \\
\hline 12 & 1423 & SE & $\beta$-Ylangeno* & $1.00 \pm 0.04 \mathrm{a}$ & $1.74 \pm 0.10 \mathrm{~b}$ & $1.80 \pm 0.05 \mathrm{c}$ & $\mathrm{p}<0.01 * *$ \\
\hline 13 & 1433 & SE & $\beta$-Copaeno & $0.52 \pm 0.03 \mathrm{a}$ & $1.50 \pm 0.10 \mathrm{~b}$ & $1.50 \pm 0.07 \mathrm{~b}$ & $\mathrm{p}<0.01 * *$ \\
\hline 14 & 1448 & SE & $\beta$-Gurjuneno & $0.50 \pm 0.02 \mathrm{a}$ & $0.63 \pm 0.05 \mathrm{~b}$ & $0.60 \pm 0.08 \mathrm{c}$ & $\mathrm{p}<0.01 * *$ \\
\hline 15 & 1459 & SE & trans- $\beta$-Farneseno & $4.61 \pm 0.26 \mathrm{a}$ & $3.70 \pm 0.08 \mathrm{~b}$ & $1.20 \pm 0.07 \mathrm{c}$ & $\mathrm{p}<0.001 * * *$ \\
\hline 16 & 1465 & SE & allo-Aromadendreno & $1.30 \pm 0.11 \mathrm{a}$ & $2.22 \pm 0.07 \mathrm{~b}$ & $2.05 \pm 0.05 \mathrm{c}$ & $\mathrm{p}<0.001 * * *$ \\
\hline 17 & 1482 & SE & $\gamma$-Muuroleno & $0.75 \pm 0.03 \mathrm{a}$ & ND & ND & - \\
\hline 18 & 1490 & SE & Biciclosesquifelandreno & $25.0 \pm 0.32 \mathrm{a}$ & $36.13 \pm 0.11 \mathrm{~b}$ & $38.0 \pm 0.08 \mathrm{c}$ & $\mathrm{p}<0.001 * * *$ \\
\hline 19 & 1499 & SE & cis- $\beta$-Guaieno & $1.22 \pm 0.11 \mathrm{a}$ & $1.50 \pm 0.08 \mathrm{~b}$ & $1.61 \pm 0.06 \mathrm{c}$ & $\mathrm{p}<0.01^{* *}$ \\
\hline 20 & 1502 & SE & $\gamma$-Amorfeno & $1.40 \pm 0.07 \mathrm{a}$ & $1.75 \pm 0.10 \mathrm{~b}$ & $1.82 \pm 0.08 \mathrm{~b}$ & $\mathrm{p}<0.01^{* *}$ \\
\hline 21 & 1517 & SE & $\gamma$-Cadideno & $0.91 \pm 0.09 \mathrm{a}$ & $1.70 \pm 0.14 \mathrm{~b}$ & $1.32 \pm 0.07 \mathrm{c}$ & $\mathrm{p}<0.01 * *$ \\
\hline 22 & 1525 & SE & $\delta$-Cadineno & $1.50 \pm 0.10 \mathrm{a}$ & $2.60 \pm 0.05 \mathrm{~b}$ & $2.34 \pm 0.08 \mathrm{c}$ & $\mathrm{p}<0.01 * *$ \\
\hline
\end{tabular}

x) M:Monoterpeno; MO:Monoterpeno oxigenado; SE:Sesquiterpeno

E1:Estadío I, E2:Estadío II, E3:Estadío III

* Identificados tentativamente

**Probabilidades significativas

***Probabilidades altamente significativas

P:probabilidad

ND:no detectado

Muñoz et al. (2007) también señalan cuatro pétalos para la flor, pero en realidad esta especie posee cinco pétalos. La confusión surge porque un par de pétalos se fusionan dando la apariencia de ser uno solo. Se evidenció que cada bráctea da soporte a una flor igual que en la Cyperaceae Cladium jamaicense (Richards 2002)

Se confirma que el desarrollo es acrópeto, lo que ocurre tanto en las ramas, como en el botón de flores. En la tercera etapa, se encuentran desde grupos de células en proceso de 
diferenciación hasta órganos florales completamente desarrollados. La bráctea se presenta desde los inicios de la ontogenia, surge sobre ella el cáliz y casi de forma simultánea la corola e inmediatamente los estambres petalostémonos. Esta asincronía en el crecimiento podría propiciar el éxito reproductivo y de variabilidad genética, pues de este modo se facilita la fertilización cruzada y además se garantiza la protección a los órganos sexuales en desarrollo (androceo y gineceo); No obstante pueden encontrarse diferencias con respecto a los tiempos en que aparecen los verticilos, la disposición de éstos y sus diferentes tamaños en otras especies.

Junto con los cambios morfológicos y anatómicos presentados durante el desarrollo de las inflorescencias de $L$. alba, se observan diferencias en la detección, aumento y disminución de los diferentes metabolitos secundarios volátiles típicos para esta especie (variación descrita en los resultados y Cuadro 1).

Estos cambios podrían deberse a que un compuesto presente, se requiera para biosintetizar otro u otros. De este modo, el precursor disminuiría o se transformaría. Según Dudareva \& Pichersky (2006), esta ausencia también podría ser causada por regulaciones existentes en los genes que codifican las enzimas biosintéticas, cambios en la información codificante (por ejemplo, mutaciones), modificaciones postraslacionales y disponibilidad del sustrato que afectan la estabilidad del producto y el proceso de esta emisión. Así mismo, la creación de nuevos compuestos esenciales podría ocurrir por mutación, la formación de nuevas enzimas y por consiguiente nuevos sustratos específicos o nuevos productos. Precisamente Bouwmeester et al (1998), reportan la síntesis de carvona a expensas del limoneno en frutos de Carum carvi L. Al parecer, en L. alba ocurre lo mismo y se observa un comportamiento inversamente proporcional en esta variación (Cuadro 1 y Fig. 7): la cantidad relativa del limoneno tiende a disminuir (4.7\% en la Etapa I, $3.8 \%$ en la Etapa II y $3.2 \%$ en la Etapa III) mientras la de la carvona aumenta $(23.6 \%$ en la Etapa I, 27\% en la Etapa II y $29.14 \%$ en la Etapa III).

De igual modo, podría darse la volatilización de los monoterpenos que depende, entre otros factores, de la temperatura, la luminosidad y la humedad. Ello es corroborado por Matile \& Altenburger (1988), Loughrin et al. (1991) y especialmente por Duarte \& Cárdenas (2007). Estos últimos autores trabajaron sólo con hojas de L. alba y tuvieron en cuenta el ciclo circadiano de la planta. Observaron que cuando se presentan condiciones de alta radiación solar y temperaturas elevadas, se obtienen contenidos altos de metabolitos secundarios, en especial de carvona, motivo por el cual se eligió el soleado medio día para los muestreos de las inflorescencias de L. alba.

También durante el proceso de extracción, varios compuestos probablemente se pierden, ya sea durante la absorción o porque la muestra concentrada es altamente volátil (Dudareva \& Pichersky 2006). Lo anterior es claramente observado al comparar los compuestos detectados en la presente investigación, con los registrados en estudios de Stashenko et al. (2004), Duarte \& Cárdenas (2007), Durán (2005), Monsalve (2007) y Agudelo \& Blanco (2007), realizados para la misma especie pero utilizando sólo hojas o de forma simultánea hojas e inflorescencias, donde aparecen más de 40 compuestos diferentes, mientras en ésta, sólo 22 fueron detectados en cantidades $>0.05 \%$; sin embargo, algunos de los mayoritarios como la carvona, el biciclosesquifelandreno y el limoneno siempre están presentes. Lo anterior también es reportado por Nickavar \& Mojab (2000), en cuya investigación se señaló a la carvona como componente mayoritario (46.7\%) seguida por el limoneno (24.6\%), para Calamintha officinalis. Se debe recordar que las diferencias en las técnicas empleadas para la extracción (Stashenko et al. 2004) y la parte de la planta (y sus estados de desarrollo), influyen marcadamente en los resultados, pues los metabolitos secundarios varían dependiendo de estas condiciones, al igual que de los diferentes lugares geográficos, épocas de recolección del 
material vegetal y variación genética (Nickavar \& Mojab 2000).

Según Dudareva \& Pichersky (2006), en los terpenos de las esencias florales el porcentaje de compuestos oxigenados es mucho más alto para monoterpenos $(81 \%)$ que para sesquiterpenos (38\%). Concordando con estos autores, de los 22 compuestos identificados en este estudio, 15 son sesquiterpenos (ninguno oxigenado), mientras que los restantes son monoterpenos y seis de ellos son oxigenados. La predominancia de sesquiterpenos fue originada probablemente por el tiempo de exposición de la fibra (PDMS/DVB), y en general, el proceso de extracción realizado; Es de resaltar que las cantidades relativas de estos últimos (monoterpenos oxigenados) represen$\tan$ un $43 \%$ del total de los volátiles obtenidos y son precisamente estos compuestos (monoterpenos y monoterpenos oxigenados), los responsables de la suave y agradable fragancia que caracteriza esta planta. Otra causa de la baja frecuencia de estas moléculas, además de su volatilidad, puede ser la ineficiente recolección por los métodos utilizados (Dudareva \& Pichersky 2006).

Un aspecto importante son las emisiones de las esencias florales que varían en el espacio, debido a que la esencia puede ser emitida desde regiones especializadas del tejido floral (Vogel 1990). A pesar de que en las flores, los pétalos son los principales emisores de volátiles, algunas veces compuestos específicos pueden ser emitidos desde un solo grupo de órganos florales (Verdonk et al. 2003). Ya se ha demostrado que, los estambres, pistilos, polen y néctar contribuyen o son los principales responsables de la emisión de ciertos compuestos. Los volátiles emitidos por el polen son notablemente diferentes a los emitidos desde otras partes de la flor (Dudareva \& Pichersky 2006). En relación a ello, se puede mencionar el $\gamma$-muuroleno que aparece sólo durante la primera etapa de desarrollo, cuando se inicia el proceso de diferenciación morfológica y anatómica de los órganos florales (siempre se detectaron los demás compuestos). Los factores genéticos juegan un papel importante en esta producción y emisión de compuestos, por ejemplo, el gen LIS es un gen que ha mostrado una alta expresión en los estigmas, con ligeros niveles en los pétalos (Dudareva et al. 1996). Así mismo, la expresión de las terpeno-sintasas en el estigma podría estar involucrada en la protección de mayor superficie de área contra el crecimiento de hongos, aunque los monoterpenos producen también actividad antimicrobial. Otra función potencial podría ser contra el estrés oxidativo (Deans \& Waterman 1993). Igualmente, la expresión del gen AtTPS es conocida para la síntesis de monoterpenos y sesquiterpenos y se ha comprobado su actividad en los tejidos florales, no obstante, los pétalos se reportan como el principal órgano de expresión de esencias no terpenoides de las esencias florales (Fiehn et al. 2000).

Continuando con la relación compuestoetapa en la presente investigación, se pueden mencionar compuestos como el $\beta$-ylangeno, el $\gamma$-amorfeno, el cis- $\beta$-guaieno y el biciclosesquifelandreno, que incrementan su porcentaje relativo durante la Etapa III e igualmente podrían estarían relacionados en su producción con los órganos florales maduros. Así mismo, se detectaron tres metabolitos diferentes a los reportados en otros estudios (Stashenko 2004, Duarte \& Cárdenas 2007, Durán 2005, Monsalve 2007, Agudelo \& Blanco 2007), con esta misma especie; ellos son: el $\beta$-copaeno, el $\gamma$-amorfeno y el cis- $\beta$-guaieno. Estos metabolitos, probablemente sean producidos sólo en la flor de esta planta (ni en hojas ni en tallos). Corroborando una vez más todo lo anterior, Shalit et al. (2003) y Lavid et al. (2002) investigaron la emisión en flores de diferentes especies con patrones de desarrollo semejantes y encontraron que ésta aumenta durante las primeras etapas de desarrollo floral, llegando al máximo cuando las flores son percibidas por los polinizadores y descendiendo después de esto. No obstante, según Euler \& Baldwin (1996), aparte de atraer polinizadores (lo cual es la función primaria de la flor), los compuestos químicos de las fragancias florales también podrían aumentar el riesgo de ataques de herbívoros a las estructuras de la flor y los 
tejidos florales pueden requerir relativamente mayor protección de enemigos. Es así como en Nicotiana attenuata se inducen en la corola metabolitos biocidas de defensa (Euler \& Baldwin 1996) y probablemente sucede lo mismo en otras plantas, incluida nuestra especie en estudio.

Las esencias de muchas flores disminuyen marcadamente poco después de la polinización. Investigaciones efectuadas con Petunia hybri$d a$, revelan que el aminoramiento en la emisión de la fragancia, empieza sólo después que el polen alcanza el ovario, sugiriendo que la polinización es un requisito para la reducción de las esencias florales luego de la polinización (Negre et al. 2003). Con ello se muestra la importante relación que existe entre los procesos del desarrollo de la planta con su ambiente y se observa que menguan compuestos tales como, cis-dihidro-carvona, trans-dihidro-carvona y trans- $\beta$-Farneseno en la Etapa III.

Es de resaltar el hallazgo del biciclosesquifelandreno como compuesto mayoritario (38\%), ya que el material vegetal de $L$. alba sometido al análisis químico, se encontraba catalogado como quimiotipo carvona. No obstante, se realizó el estudio sólo con las inflorescencias de esta planta (el primer estudio conocido de este tipo); probablemente, de haber sido realizado también el análisis a las hojas, éstas, como siempre ha sucedido para el cultivo experimental del cual se tomaron las muestras, hubiesen registrado a la carvona como analito principal.

Otras funciones de los metabolitos secundarios incluyen la defensa y protección contra o durante el estrés abiótico (Euler \& Baldwin 1996) y la protección de zonas con heridas después de la abscisión de los órganos florales (Dudareva \& Pichersky 2006). Estas funciones adicionales pueden ayudar a explicar la abundancia y variedad de los diferentes analitos detectados (Euler \& Baldwin 1996).

El conocimiento de las esencias florales es un aspecto crítico para el entendimiento de las funciones evolutivas y biológicas. El progreso de la investigación en esta área resulta un aporte significativo al conocimiento farmacognóstico de esta especie vegetal medicinal, además de constituir una fuente de apoyo para biólogos, bioquímicos, químicos analíticos y de síntesis (Dudareva et al. 2000). Más aún resulta importante si se considera que las reconstrucciones filogenéticas de las plantas basadas en los compuestos esenciales, son aún escasas (Azuma $e t$ al. 1999).

Con los resultados de este estudio, se describen caracteres poco conocidos del desarrollo floral de $L$. alba, además de reportar compuestos que al parecer son exclusivos de la fragancia (el $\beta$-copaeno, el $\gamma$-amorfeno y el cis- $\beta$-guaieno). Se confirmó que ocurren cambios a nivel químico durante la ontogenia de las inflorescencias, hecho que seguramente acompaña el desarrollo de los órganos sexuales de otras especies vegetales.

\section{AGRADECIMIENTOS}

Los autores expresan sus más sinceros agradecimientos a María Nilda Cerf Arbulú y a José Miguel Esborronda Andrade, por la contribución con sus conocimientos y sugerencias para la realización de este proyecto y para todas las áreas del Saber. A la Federación Internacional de Sociedades Científicas (FISS) por el material bibliográfico. Agradecemos igualmente a Jhon Díaz Olarte por su apoyo en el diseño y revisión estadística, a Nestor Marín por sus asesorías en la parte química, a Humberto García Pinzón por sus aportes en la descripción de las inflorescencias y a Edgar Rincón Barón, por contribuir con su experiencia en Histotécnia, al desarrollo de esta investigación.

\section{RESUMEN}

La especie Lippia alba (Verbenaceae) llama la atención de los investigadores por su amplia distribución en todo el mundo y sus diversas propiedades. Se establecieron tres etapas durante el desarrollo de sus inflorescencias, fueron recolectadas en Bucaramanga, Colombia para analizar los cambios morfoanatómicos. Para ello, las muestras se trataron mediante la técnica safraninafastgreen y se observaron utilizando microscopio óptico convencional. Se realizó el aislamiento de los metabolitos 
secundarios volátiles usando la técnica de microextracción en fase sólida en el modo headspace (HS-SPME) y su posterior análisis de componentes, mediante cromatografía de gases acoplada a espectrometría de masas (GC-MS). En la Etapa I se observó una masa de células meristemáticas en el ápice vegetativo. En la Etapa II la acción de procesos morfogenéticos dan paso a la formación de los verticilos florales del perianto. En la Etapa III se establecen todos los verticilos florales. Los principales metabolitos secundarios constituyen el $78 \%$ de la cantidad relativa total. Ellos son el biciclosesquifelandreno, la carvona, el limoneno y el trans- $\beta$-farneseno. Los metabolitos $\beta$-copaeno, $\gamma$-amorfeno y cis- $\beta$-guaieno, no están registrados en la bibliografía revisada. Estos compuestos activos pueden variar de acuerdo con el estado de desarrollo de la planta, la parte empleada para la extracción y el método implementado para ello.

Palabras clave: Lippia alba, Verbenaceae, inflorescencias, ontogenia, morfología y anatomía floral, metabolitos secundarios volátiles.

\section{REFERENCIAS}

Adams, R.P. 1995. Identification of essential oil components by gas chromatography / mass spectrometry. Allured, Carol Stream, Illinois, EEUU.

Agudelo, A \& K. Blanco. 2007. Estudio comparativo de los aceites esenciales de Lippia alba Mill N.E. Brown ex Britton \& Wills cultivada con tres tipos de compostaje. Tesis de Grado. Universidad Industrial de Santander, Bucaramanga, Colombia.

Azuma, H., L.B. Thien \& S. Kawano. 1999. Molecular Phylogeny of magnolia (Magnoliaceae) inferred from cpDNA sequences and evolutionary divergence of the Floral Scents. J. Plant. Res. 112: 291-302.

Bandoni, A. 2002. Los recursos vegetales aromáticos en Latinoamérica, su aprovechamiento industrial para la producción de aromas y sabores. CYTED, Buenos Aires, Argentina.

Bouwmeester, H.J., J. Gershenzon., M. Konings \& R. Croteau. 1998. Biosynthesis of the monoterpenes limonene and carvone in the fruit of caraway. Demonstration of enzyme activities and their changes with development. Am. Soc. Plant. Biol. 117: 901-912.

Carvalho, C \& M. Fonseca. 2005. Carvone: Why and how should one bother to produce this terpene. Food Chem. 95: 413-422.

Cerana, M. 2004. Flower morphology and pollination in Mikania (Asteraceae). Flora. 199: 168-177.
Deans, S.G \& P.G. Waterman. 1993. Biological activity of volatile oils, in volatile oil Crops: Their biology, biochemistry and production. Long. Scient, Nueva York, EEUU.

Dong, Z., Z. Zhao, C. Liu, J. Luo, J. Yang \& W. Huang. 2005. Floral Patterning in Lotus japonicus. Plant Physiol. 137: 1272-1282.

Duarte, S., C. Cárdenas, J. Martínez \& E. Stashenko. 2007. Estudio de la variación circadiana de los metabolitos secundarios volátiles obtenidos por la destilación extracción con solvente simultánea de hojas de Lippia alba (fam. Verbenaceae). Scientia et Technica 13: 83-85.

Dudareva, N. \& E. Pichersky. 2006. Biology of floral scent. Taylor \& Francis Group, Nueva York, EEUU.

Dudareva, N., B. Piechulla \& E. Pichersky. 2000. Biogenesis of floral scent. Hort. Rev. 24: 31-54.

Dudareva, N., L.Cseke, V.M. Blanc \& E. Pichersky. 1996. Evolution of floral scent in Clarkia: novel patterns of s-linalool synthase gene expression in the $C$. breweri flower. Plant Cell. 8: 1137-1148.

Durán, C. 2005. Estudio del aceite esencial de Lippia alba (Fam. Verbenaceae) y de los aspectos fisiológicos en diferentes etapas de su crecimiento bajo tres niveles de luz. Tesis de Grado, Universidad Industrial de Santander, Bucaramanga, Colombia.

Euler, M. \& I.T. Baldwin. 1996. The Chemistry of defense and apparency in the corollas of Nicotiana attenuata. Oecologia 107: 102-108.

Evans, R. \& T. Dickinson. 1999. Floral ontogeny and morphology in subfamily Spiraeoideae Endl. (Rosaceae). Int. J. Plant Sci. 160: 981-1012.

Fiehn, O., J. Kopka, P. Dormann, T. Altmann, R.N. Trethewey \& L. Willmitzer. 2000. Metabolite profiling for plant functional genomics. Nat. Biotechnol. 18: 157-168.

García, H. 1975. Plantas medicinales de Colombia. Tomo II. Universidad Nacional de Colombia, Bogotá, Colombia.

Gupta, M. 1995. 270 plantas medicinales iberoamericanas. CYTED-SECAB, Bogotá, Colombia.

Gurgel Do Vale, T., J. Couto, Jr. Santos \& G. Viana. 2002. Central effects of citral, myrcene and limonene, constituents of essential oil chemotypes from Lippia alba (Mill.) N.E. Brown. Phytomed. 9: 709-714. 
Guzmán, S., R. Cardozo \& V. García. 2004. Desarrollo agrotecnológico de Lippia alba (Miller) N.E.Br ex Britton y Wilson. Guillermo de Ockhman 7: 201215.

Johansen, D. 1940. Plant microtechnique. McGraw-Hill, Nueva York, EEUU.

Joulain, D. \& W. König. 1998. The atlas of spectral data of sesquiterpene hydrocarbons. E.B, Verlag, Hamburgo, Alemania.

Kovàts, E. 1965. Gas chromatographic characterization of organic substances in the retention index system. Adv. Chromatogr. 1: 229-247.

Krizek, B. \& J. Fletcher. 2005. Molecular mechanisms of flower development: an armchair guide. Nature 6: 688-698.

Kumar, S. \& J. Bahl. 2002. Lippia alba, planta llamada "Bhurakshak". United States Plant Patent. Patent No: US PP13, 110 P2. (Consultado 22 diciembre 2008, www.books.google.com/patents?id=Bhurakshak).

Lavid, N., J. Wang, M. Shalit \& I. Guterman. 2002. O-methyl transferases involved in the biosynthesis of Volatile Phenolic derivates in rose petals. Am. Soc. Plant. Biol. 129: 1899-1907.

Loughrin, J.H., D.A. Potter \& T.R. Hamilton-Kemp. 1991. Circadian rhythm of volatile emission from flowers of Nicotiana sylvestris and N. suaveolens. Physiol. Plant 83: 492-496.

Matile, P. \& R. Altenburger. 1988. Rhythms of fragance emission in flowers. Planta 174: 242-247.

Monsalve, L.A. 2007. Comparación de la composición química de los aceites esenciales de las plantas de Lippia alba (Mill) N.E Brown, provenientes de diferentes regiones de Colombia. Tesis de grado, Universidad Industrial de Santander, Bucaramanga, Colombia.

Mora, L. 1987. Estudios morfológicos, autoecológicos y sistemáticos en Angiospermas. Rev. Acad. Col. Cien. Exact. Fis. Nat.1: 19-54.

Múlgura de Romero, M., S. Martínez, A. Suyama. 1998 Morfología de las Inflorescencias en Lippia (Verbenaceae). Darwiniana 36:1-12.

Muñoz, A., F. Vallejo \& M. Sánchez. 2007. Morfología y anatomía de las flores y semillas de pronto alivio. Acta Agron. Col. 56: 7- 11.

Negre, F., C.M. Kish, J. Boatright, B. Underwood, K. Shibuya, C. Wagner, D.G. Clark \& N. Dudareva. 2003. Regulation of methylbenzoate emission after pollination in snapdragon and petunia flowers. Am. Soc. Plant. Biol. 15: 2992-3006.

Nickavar, B. \& F. Mojab. 2000. Hydrodistilled volatile constituents of Calamintha officinalis Moench from Iran. JEOBP 8: 23-27.

Ocampo, R. \& R. Valverde. 2000. Manual de cultivo y conservación de plantas medicinales. Tomo I. Instituto de Investigaciones Fundamentales en Agricultura Tropical, Tramil, San José, Costa Rica.

Oliveira, D., S. Santos, H. Bizzo \& D. Alviano. 2006. Ethnopharmacological study of two Lippia species from Oriximiná, Brazil. J. Ethnopharm. 108: 103-108.

Pascual, M., K. Slowing, E. Carretero, D. Sánchez \& A. Villar. 2001. Lippia: Traditional uses, chemistry and pharmacology: a review. J. Ethnopharmacol. 76: 201-214.

Perreta, M. \& A. Vegetti. 2004. Estructura de las inflorescencias en especies de Melica (Meliaceae, Pooideae, Poaceae). Darwiniana 42: 37-49.

Pino, J., L. Ariel, A. Pérez \& M. Rodríguez. 1996. Composición y propiedades antibacterianas del aceite esencial de Lippia alba (Mill.) N. E. Brown Rev. Cubana Farm. 30: 1-3.

Richards, J.H. 2002. Flower and spikelet morphology in sawgrass, Cladium jamaicense Crantz (Cyperaceae). Ann. Bot. 90: 361-367.

Ricciardi, G., A. Ricciardi., A. Bandoni. 2000. Fitoquímica de Verbenáceas (Lippias y Aloysias) del Nordeste Argentino. Comunicaciones Científicas y Tecnológicas, Universidad Nacional del Nordeste, Corrientes, Argentina.

Rueda, R. 1994. Systematics and evolution of the genus Petrea (Verbenaceae). Ann. Mo. Bot. Gard. 81: 610-652.

Shalit, M., I. Guterman, H. Volpin, E. Bar, T. Tamari, N. Menda, Z. Adam, D. Zamir, A. Vainstein \& D. Weiss. 2003. Volatile ester formation in roses. Identification of an acetyl-coenzyme a geraniol /citronellol acetyltransferase in developing rose petals. Plant Physiol. 131: $1868-1876$

SPSS para Windows. 2004. Versión 13.0. Chicago: SPSS Inc. Programa informático en CD-ROM. [También disponible en línea: SPSS Inc: http://www.spss. $\mathrm{com} /]$

Stashenko, E., B. Jaramillo \& J. Martínez. 2004. Comparison of different extraction methods for the analysis of volatile secondary metabolites of Lippia alba (Mill.) 
N.E. Brown, grown in Colombia, and evaluation of its in vitro antioxidant activity. J. Chromatogr. 1025: 93-103.

Vale, T., F. Matos \& G. Viana. 1999. Behavioral effects of essential oils from Lippia alba (Mill.) N.E. Brown chemotypes. J. Ethnopharm. 167: 127-133.

Verdonk, J.C., C.H. Ric de Vos, C.H. Verhoeven, H.A. Haring, M.A. Van Tunen \& R.C. Schuurink. 2003. Regulation of floral scent production in Petunia revealed by targeted metabolomics. Phytochemistry 62: 997-1008.

Vogel, S. 1990. The role of scent glands in pollination: on the structure and function of osmophores. Smi- thsonian Institute Libraries, Amerindl, Nueva Delhi, India.

Vit, P., B. Silva \& P. Meléndez. 2002. Lippia alba N.E.Br. Ficha botánica de interés apícola en Venezuela, No. 2 Cidrón. Rev. Fac. Farm. 43: 13-14.

Yamamoto, P. 2006. Interação genótipo X ambiente na produção e composição de óleos essenciais de Lippia alba (Mill.) N. E. Br. Tesis de posgrado, Instituto Agronómico Campinas, São Paulo, Brazil.

Zétola, M., T. De Lima, G. Sonaglio, R. González, P. Limberger, P. Petrovick \& V. Bassania. 2002. CNS activities of liquid and spraydried extracts from Lippia alba Verbenaceae (Brazilian false melissa). J. Ethnopharm. 82: 207- 215. 